\title{
Vital capacity in tetraplegics twenty years and beyond
}

\author{
AMP-E Tow ${ }^{*}, 1$, DE Graves ${ }^{2}$ and RE Carter ${ }^{2}$ \\ ${ }^{1}$ Department of Rehabilitation Medicine, Tan Tock Seng Hospital, C/o Ang Mo Kio Community Hospital, Republic \\ of Singapore; ${ }^{2}$ Department of Physical Medicine and Rehabilitation, Baylor College of Medicine, The Institute of \\ Rehabilitation and Research, Houston, Texas 77030, USA
}

Objectives: To observe the trends in vital capacity (VC) over time in tetraplegics 20 years and
more after injury, the effects of age at injury, severity of injury and gender on this trend.
Methods: The medical records of all spinal cord injured persons admitted to a regional spinal
injury center from January 1960 to December 1996 were reviewed. Fifty-seven patients had
documented post-rehabilitation VC (mean $1.3 \pm 1.1$ years) and VC at 10 (mean 11.8 2.69 )
and $20(20.60 \pm 2.67)$ years post injury and beyond. Results: The mean age at injury was $23.2 \pm 9.1$ years. Severity of injury when classified according the system proposed by Coll et al were: Group 1: C1 - 4 Frankel A injury: $11.6 \%$, Group 2: C5-8 Frankel A injury: 55.6\%, Group 3: C2-8 Frankel B and C: $29.8 \%$ and Group 4: C2-8 Frankel D: 3.5\% respectively. The mean VC at initial, 10 and 20 years post injury was $2586 \pm 948,2803 \pm 940$ and $2525 \pm 818 \mathrm{cc}$ respectively. Multivariate analysis of variance revealed that there was significant difference in VC over a 20 year period, $(\mathrm{F}(2,54)=8.43, P<0.05)$. The difference between $\mathrm{VC}$ at 10 years and $\mathrm{VC}$ at 20 years accounted for the $19.8 \%$ of the variance in $\mathrm{VC}$ over time $(\mathrm{F}(1,55)=12.35, P<0.05)$. Age at injury, gender and severity of injury did not have a significant influence on the rate of decline in VC. Analysis of a subset of 26 patients who were followed up more than 20 years post injury (range 22 to 34.5 years) revealed similar, with a greater drop in the VC from 10 years post injury $(\mathrm{F}(1,23)=6.52, P<0.05)$. In this subset of patients, the mean VC at initial injury was $2840.9 \pm 847.3 \mathrm{cc}$, at 10 years was $2549.6 \pm 750.3 \mathrm{cc}$, at 20 years was $2400.9 \pm 724.1 \mathrm{cc}$ and beyond 20 years was $2194.2 \pm 738.7 \mathrm{cc}$. There was no significant difference in mean VC between non smokers and ex/current smokers at initial, 10 and 20 years post injury, using the independent $t$-test $(P>0.05)$.

Conclusion: Vital capacity in tetraplegics declines significantly over the years, with a greater decline occurring at more than 20 years post injury.

Spinal Cord (2001) 39, $139-144$

Keywords: vital capacity; tetraplegia; spinal cord injury; respiratory; aging

\section{Introduction}

It has long been noted that patients with cervical spinal cord injury (SCI) have an alteration in their breathing mechanics because of the abnormally high extrapulmonary work of breathing attributable to the use of the diaphragm as the main source of ventilation. ${ }^{1}$ This is demonstrated clinically by paradoxical retraction of the ribcage upon contraction of the diaphragm, especially so in those with absent inspiratory scalene activity. ${ }^{2}$ As pulmonary complications represent a major threat to the lifespan of tetraplegics especially those with complete lesions at any level of the cervical cord, periodic and frequent measurement of ventilatory status is helpful. Historically, vital capacity (VC) has been used as a bedside measure of ventilation in both

*Correspondence: AMP-E Tow, Department of Rehabilitation Medicine, Tan Tock Seng Hospital, C/o Ang Mo Kio Community Hospital, 17 Ang Mo Kio Ave 9, Singapore 560766, Republic of Singapore acutely ill and chronic SCI patients. Vital capacity is defined as the maximal volume of air that can be exhaled from the lungs following a maximal inspiration. As a gross measure of overall pulmonary function, the evaluation of vital capacity can be useful in determining a patient's ability to maintain adequate gas exchange without undue work of breathing. Unlike other more complicated testing methods, VC can be measured using a convenient, easily transportable hand held device. Potential benefits of using VC measurements in SCI patients include earlier detection of respiratory compromise and improved monitoring of clinical progress. Roth has shown that the $\mathrm{VC}$ was found to be significantly correlated with forced expiratory volume in $1 \mathrm{~s}$, inspiratory capacity, expiratory reserve volume, functional residual capacity, residual volume, total lung capacity, and RV/TLC ratio in acute SCI patients. ${ }^{3}$ Over the years, it has been utilized for regular and frequent documentation of 
gross pulmonary function in SCI patients managed at The Institute for Rehabilitation and Research (TIRR). The purpose of the current investigation is to investigate the trend in $\mathrm{VC}$ in tetraplegics over the post injury years and to observe the effects of severity of injury, age at injury and gender on this trend in vital capacity.

\section{Methods}

\section{Subjects and materials}

The charts of all traumatic SCI tetraplegic patients on follow up at TIRR since 1960 were reviewed. Only those patients who had been followed up for at least 20 years and who had documented VC at initial visit and at 20 years with at least one $\mathrm{VC}$ measurement in between, were included in the study. The time of initial VC recorded ranged from September 1961 to July 1979. As far as possible, VC measurements were documented in every patient at initial admission/evaluation at TIRR, and at every outpatient follow-up. Patients were seated in the upright position when $\mathrm{VC}$ was measured. A hand held Wright Respirometer was used for every VC reading. The equipment and methodology of obtaining the $\mathrm{VC}$ follows closely the American Thoracic Society recommendations for measuring Forced Vital Capacity. ${ }^{4}$ The patients were instructed to exhale with maximally forced effort from a position of maximal inspiration. In order to reduce the error that may be introduced with different persons conducting the VC measurement over the years, the method of measuring VC was closely and individually supervised by the same attending physician throughout the years and during the period of the study. The best reading out of three trials is taken as the $\mathrm{VC}$ reading. The $\mathrm{VC}$ at initial injury was taken to be that value when the VC has been stabilized from acute injury, for example, at the end of the rehabilitation stay. This is because VC is known to increase by as much as $100 \%$ up to a few months post injury. ${ }^{5}$ Likewise, as values at exactly 10 and 20 years post injury were not always available, the $\mathrm{VC}$ taken at the times closest to 10 (mean $11.81 \pm 2.69$ ) and 20 (mean $20.61 \pm 2.67$ ) years post injury were taken. For purposes of obtaining an accurate smoking history, all patients were interviewed via the telephone.

\section{Procedure/statistical analysis}

The demographic variables are described in descriptive statistics. Repeated measures analysis of variance was performed in data from 57 cases using the initial VC and that at approximately 10 and 20 years post injury. In order to investigate whether VC dropped significantly after 20 years of injury, a second repeated measures analysis of variance was performed on a subset of 26 of those cases that had a fourth VC measurement at more than 20 years post injury. The
$\mathrm{VC}$ readings of patients were used as their own controls in respect to comparing the VC measurements over time.

The patients were classified using the classification described by Coll in order to provide three patient groups for the repeated analysis of variance. The Coll classification taken into account the level and extent of injury, according to the ASIA scale. Coll group 1 includes $\mathrm{C} 1-4$ Frankel A tetraplegia, Coll group 2: C5-8 Frankel A tetraplegia, group 3: all individuals with Frankel B and C tetraplegia and Coll group 4 includes all individuals with Frankel D tetraplegia. ${ }^{6}$ For this investigation, Coll groups 3 and 4 were combined to give a total of three groups, as the number of patients in group 4 was too small for proper analysis $(n=2)$. The measurement at three periods in time provided a 3 by 3 matrix using the vital capacities as dependent measures for this analysis.

\section{Results}

\section{Demographic characteristics}

Seventy-two per cent of patients were male, and $22.8 \%$ female. The majority were injured from diving injuries $(33.3 \%)$, followed by motor vehicle crashes $(31.6 \%)$, sport injuries $(14.0 \%)$ and falls $(8.8 \%)$. Age at injury ranged from 14 to 56 years, with a mean of $23.2 \pm 1.2$ years, and median of 20.0 years. Injuries at the $\overline{C 5}-6$ levels were the commonest $(66.7 \%)$ followed by $\mathrm{C} 7-8$ injuries $(19.3 \%)$ and $\mathrm{C} 2-4$ injuries $(14.1 \%)$. This proportion is fairly representative of the patients seen at TIRR, which had approximately $16.4 \%$ sustaining injuries at $\mathrm{C} 1-4$ and $30.2 \%$ injuries at $\mathrm{C} 5-8$ in an analysis done in $1996 .^{7}$ Sixty-five per cent sustained complete Frankel A injuries. When classified according to the classification as proposed by Coll, the majority of patients consisted of Coll group 2 patients, ie, C5-8 complete tetraplegics $(55.6 \%)$ followed by Coll group 3: all level cervical Frankel B and C (29.6\%) and Coll group 1: $\mathrm{C} 1-4$ Frankel A (11.6\%) respectively. Although one patient had cervical injury at C2 level, it was not complete.

Smoking history obtained through telephone interviews conducted at the time of the study revealed that there were altogether 23 non smokers $(40.4 \%)$, six smokers $(12.3 \%), 18$ ex smokers $(31.6 \%)$. Of these, all six current smokers were males. Smokers were defined as those who were still smoking at the time of interview. Ex smokers were defined as those who stopped smoking after the injury.

\section{Medical complications}

Twenty-one per cent required tracheotomy from prolonged ventilation during hospital stay. Over the years, $2 \%$ of patients developed tracheal stenosis, $3.5 \%$ developed bronchitis/pneumonia requiring hospitalization, $5.3 \%$ developed sleep apnea, of which one patient 
required ventilation, namely continuous positive airway ventilation.

\section{Vital capacity trend over time}

For the 57 patients with VC's at initial, 10 and 20 years post injury, the mean VC at injury, 10 and 20 years post injury was $2586 \pm 948,2803 \pm 940$ and $2525 \pm 818 \mathrm{cc}$ respectively (Figure 1 ). The mean time from injury that the $\mathrm{VC}$ was taken at initial, 10 and 20 years post injury was $1.3 \pm 1.1$ years, $11.8 \pm 2.6$ years and $20.6 \pm 2.6$ years respectively. Males had a significantly higher mean $\mathrm{VC}$ at initial, 10 and 20 years post injury compared to females. The rate of decline in VD did not differ significantly between the groups. There was no significant difference in VC between the patient groups classified according to the Coll classification, but there was a significant difference over time for all the patient groups $(\mathrm{F}(2,53)=3.70$, $P<0.05)$. Even though the patient groups demonstrated difference in $\mathrm{VC}$, their rate of decline did not differ significantly. Age at injury was used as a covariate to control for the effects of the aging process on the VC. It was not found to be significant.

For the subset of patients with VC documented at initial, 10, 20 and more than 20 years post injury, the mean VC at initial injury was $2840.9 \pm 847.3 \mathrm{cc}$, at 10 years was $2549.6 \pm 750.3 \mathrm{cc}$, at 20 years was $2400.9 \pm 724.1 \mathrm{cc}$ and beyond 20 years was $2194.2 \pm 738.7 \mathrm{cc}$ (Figure 2). The mean time that VC was taken at more than 20 years post injury was $26.2 \pm 3.6$ years. There was a statistically significant decline over time in all three Coll groups indicating that there was a change in $\mathrm{VC}$ over time $(\mathrm{F}(3,21)=3.12, P<0.05)$. Contrast of the individual group means shows that the decline is found when comparing $\mathrm{VC}$ measured at more than 20 years post injury to those $\mathrm{VC}$ measured previously (ie, initial, 10 and 20 years $)(\mathrm{F}(1,23)=6.52, P<0.05)$. Results of the repeated analysis of variance showed that although there was a tendency for Coll group 1 patients to consistently have lower VC at all three times, this difference did not reach statistical significance.

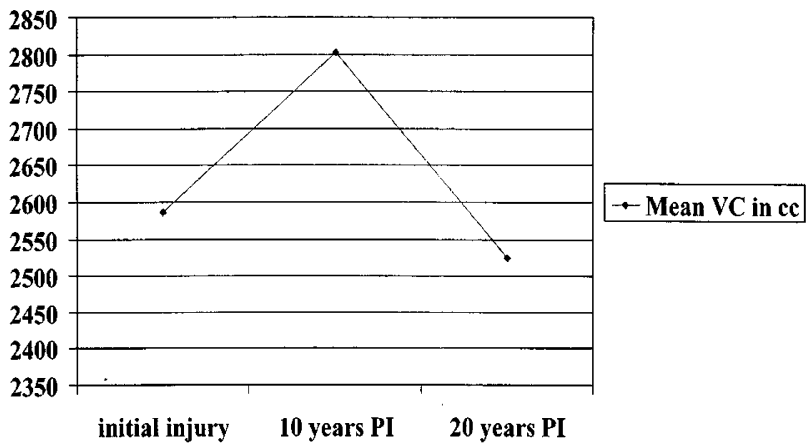

Figure 1 Mean VC at initial injury, 10 and 20 years post injury

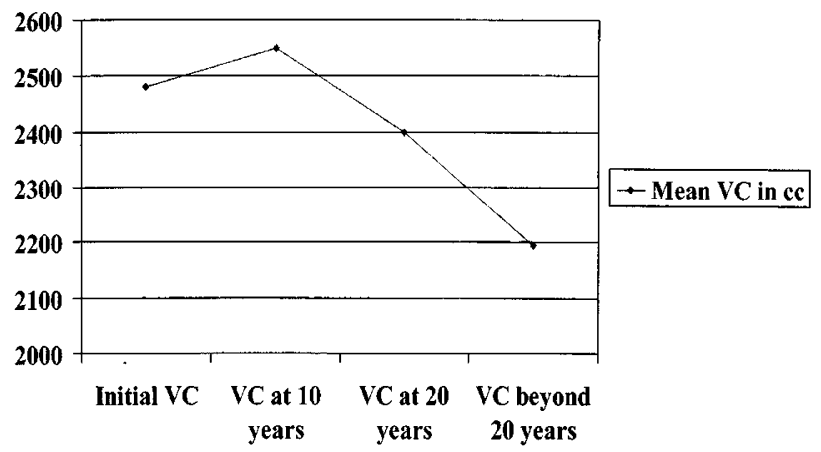

Figure 2 Mean VC for subset of 27 patients at initial 10, 20 years and beyond 20 years post injury

\section{Influence of smoking}

The VC of smokers and non smokers/ex smokers were compared using the independent $t$-test. There was no significant difference between the $\mathrm{VC}$ of current and non/ex smokers at initial $(2640.5 \pm 961.6$ vs $2611.4 \pm 2206.5 \mathrm{cc}), \quad 10 \quad(2805.4 \pm 940.0$ vs $2800.0 \pm$ $1067.7 \mathrm{cc})$ and 20 years post injury $(2489.5 \pm 741.9 \mathrm{vs}$ $2514.3 \pm 1292.9 \mathrm{cc})(P>0.1)$. In view of the fact that all current smokers in the study were males, the independent $t$-test was repeated between smokers and ex/non smokers in only the male patients in the study $(n=40)$. This similarly revealed that there was no significant difference in $\mathrm{VC}$ between non smokers and ex/current smokers for the VC at initial, 10 and 20 years post injury $(P>0.05)$.

\section{Discussion}

Cervical spinal cord injury can result in paralysis of respiratory muscles whose innervating roots emerge below the level of transection. With increasing standards of medical care, more tetraplegic individuals are surviving into the advanced years, although cardiopulmonary complications continue to plague these individuals both during the acute phase and during later years. ${ }^{8}$ Patients with complete high cervical injuries between $\mathrm{C} 1-2$ levels, especially after the initial edema subsides, would be expected to be apnoeic and require long term respiratory support in terms of either mechanical ventilation or electrophrenic respiration. ${ }^{9}$ Patients with mid or lower cervical cord injuries with levels at $\mathrm{C} 3$ to $\mathrm{C} 8$, as is the majority of patients in our study, are left with variable degrees of respiratory dysfunction, especially with respect to pulmonary mechanics. These individuals whose phrenic nerves are completely or partially intact can contract their diaphragms to a variable extent. However, paralysis of the abdominal muscles as well as inspiratory intercostal and most expiratory muscles results in altered rib cage motion with the abdominal wall pushed out and the lower ribs drawn during inspiration, generating a paradoxical pattern in rib cage movement. ${ }^{10}$ Despite electromyographic evidence of large amounts of phasic 
inspiratory activity of the accessory muscles of respiration, paradoxical inward displacement of the lower rib cage occurs, causing a marked reduction in vital capacity. ${ }^{11}$ In addition, paralysis of all the intercostal muscles causes major changes in rib cage compliance, ${ }^{12}$ even though specific lung compliance, which is compliance corrected for lung volumes, is normal. ${ }^{13}$ The loss of use of the abdominal and other expiratory muscles from coughing and clearing secretions, placing them at a higher risk of respiratory tract infections. ${ }^{14}$ However, coughing can be partially preserved in lower cervical injuries, as it has been shown that the clavicular head of the pectoralis can provide some expiratory activity, being innervated from the fifth through seventh cervical segments. ${ }^{15}$

Through the years the impact of aging on the musculoskeletal and respiratory systems will affect respiratory function. In our study, although there was no patient with obvious kyphoscoliosis, subtle structural changes in the spine may occur as kyphoscoliosis of the thoracic spine increases with age. In addition, costal cartilage may become calcified. These changes collectively reduce chest wall compliance and increase dependence on abdominal and diaphragmatic breathing. ${ }^{16}$ In the distal airways of older patients, alveolar ducts increase in size and volume, due to a remodeling of alveolar septa with decreased collagen and reticulum fibers results in a decrease in the surface area of the lung available for respiration. Tracheal and central airway diameter also increases with age, increasing the functional anatomical dead space. ${ }^{17}$

The documented VC should reflect the direction of change in overall lung function of the individual. Grimm found a less than $6 \%$ variability on average in spirometry measurement in SCI patients, similar to that observed in normal subjects. ${ }^{18}$ It is interesting to note that the trend in vital capacities across the patient groups showed a similar pattern in which there was an initial rise, leading to a peak at about 10 years and then a decline in $\mathrm{VC}$. The follow-up $\mathrm{VC}$ of the majority of patients in our study were taken in an outpatient setting, and the rise in $\mathrm{VC}$ over time occurred in our study population with the patients continuing in their usual activities of daily living at home.

It is well known that inspiratory muscle function can recover late in the post injury period. ${ }^{19,20}$ Earlier studies imply that only with progressive resistance exercise in already functional muscles does one find reports of a significant increase in muscle strength or VC in the years following injury. ${ }^{21,22}$ Carter noted in his study on experiences with high tetraplegics that patients admitted several months after their injury were still capable of significant gains in their VC after an inpatient rehabilitation program using appropriate respiratory exercises and therapy. ${ }^{23}$

Hoffman compared the lung function values of sedentary tetraplegic patients with that of 'athletic' SCI patients. The 'athletic' group had higher values for $\mathrm{VC}$ and for maximum ventilation. The improved respiratory condition in the athletic group was attributed to the general training effect on the ventilatory muscles. This form of training was nonspecific consisting of general activities which required increased inspiratory efforts. ${ }^{24}$ Although the influence of different activity levels was not studied in detail in our study population, the majority of our tetraplegic patients did not undertake regular aerobic exercise or vigorous sporting activity.

Motor function has been noted to stabilize within 3-4 months of injury, ${ }^{22}$ and inspiratory capacity has been noted to increase by $47 \%$ over acute post injury levels by 1 year post injury. ${ }^{25}$ The findings in our study show that VC can increase within and up to 10 years post injury without any structured respiratory program. Only two other studies documented late VC increases without a structured respiratory program. One study reported a population of 31 tetraplegics who regained autonomous breathing function a mean of $2.4 \pm 2.2$ years (1 month to 8 years) following injury. ${ }^{26}$ Another study on pulmonary function in tetraplegics documented a $49 \%$ increase in $\mathrm{VC}$ in the supine position and $60 \%$ increase in the sitting position occurring over a mean period of 7.7 to 11.6 years after injury without any specific treatment. ${ }^{27}$ This implies that inspiratory muscle strength and possibly the VC's of some patients may be still improving years after injury. As the initial reduction in $\mathrm{VC}$ in patients with spinal cord injury is out of proportion to what would be expected from muscle weakness alone, ${ }^{28}$ possible causes could be an improvement in microatelectasis following the acute period, gradual adaptation to the increased ventilatory requirements over the years, or increased spasticity leading to splinting of the lower thoracic rib cage. In addition, improved diaphragmatic strength and endurance following the loss of the intercostals and abdominal muscles in tetraplegics could be contributory. These interesting findings need to be further documented in prospective studies.

It is understandable that $\mathrm{VC}$ can decline through the years. In physically intact individuals, VC plateaus at 19 years of age and then decreases at a rate of $1-$ $1.2 \%$ per year. ${ }^{29}$ In patients with neuromuscular disease, $\mathrm{VC}$ has been shown to decline at an ever faster rate. ${ }^{30}$ In our study, an obvious decline in VC occurred after approximately 10 years post injury. Until only recently, we have assumed that SCI does not have any intrinsic influence upon the rate of aging. In a sense, SCI may be viewed as a form of instant aging. ${ }^{31}$ With paralysis comes loss of sensation, loss of movement, loss of bone density, loss of bowel and bladder function, and other physiologic changes. The years following spinal cord injury may be associated with acceleration of the aging process because of the diminished reserves, and because of the increased demands made on the still functioning bodily systems. Due to the total immobility of the lower body and the loss of sympathetic innervation, some aspects of functional deterioration may in fact proceed faster 
after SCI. ${ }^{31}$ However, to date, it is not certain whether the functional loss from SCI merely adds to the normal aging process, or has an accelerating effect. Whiteneck and Charlifue report that at about 30 years post injury, typically after 50 years of age, the individual may move from a phase of health maintenance into a phase of premature decline. After many years of confinement to a wheelchair, it has been observed that skin breakdowns become even more frequent and pulmonary complication result from a decreased respiratory reserve. In addition, obesity has a negative impact on many organ systems. ${ }^{32}$ In our study, the cohort of patients followed up more than 20 years post injury demonstrated an even steeper decline in the VC after 20 years post injury. No cohort of SCI has yet been investigated over decades to determine the impact of aging on pulmonary function or the modification of such aging by a regular exercise program. Nonetheless, there is data to show that exercise can prevent a decrease of various physiological functions in SCI individuals. As long as organ function is maintained by an active lifestyle, it is likely to delay deterioration due to the aging process. ${ }^{24}$

Age at injury was not found to be a significant covariate on the $\mathrm{VC}$ trend or rate of decline over the years. This does not conform to the assumption that the older the individual is at the time of SCI onset, the less resilient he or she may be in adapting to the injury. However, it should be noted that in our study, there is a clustering effect with the majority of patients injured in their twenties and hence the contribution of patient's age at injury may not be obvious in the statistical analysis.

Type of neurologic injury and completeness of the injury may create very different patterns of change within the aging process. This was not shown to be so in our study population, with most patients in different Coll groups showing similar rates of decline in VC. The parallel decline in $\mathrm{VC}$ in all four Coll groups over the years implies a similar process in all four groups contributing to the decline, even though they differed in terms of the degree of respiratory muscle involvement as well as completeness of injury.

With respect to medical complication, one patient had tracheal stenosis $(1.8 \%)$ in our study. Hsu reported an incidence of $1.2 \%$ of glottic and/or tracheal stenosis in a 21-year retrospective study of SCI patients admitted to their hospital. ${ }^{33}$ Tracheal stenosis is more poorly tolerated in the spinal cord injured who has compromised respiratory function. Suggested improvements in management include avoidance of prolonged endotracheal intubation, performance of tracheostomy using standard tracheostomy technique to preserve integrity of the cartilage, controlling infection, avoiding high tracheostomy at or above the second tracheal ring and frequent monitoring of tracheostomy cuff pressures. ${ }^{33}$ Pneumonia continues to plague the SCI individual, emerging as one of the leading causes of death even in the post rehabilitation period. ${ }^{34}$ This is especially true in complete tetraplegic and paraplegic patients. It is interesting to note that the number of episodes of pneumonia requiring hospitalization in our study population was relatively low. This could be the effect of under documentation, ie, the patient could have been admitted to another hospital and hence the episode not recorded. Similarly, the incidence of sleep apnea in our study population is slightly less $(5.3 \%)$ than reported in other tetraplegic populations, with the reported incidence ranging from $15 \%$ to $30 \%{ }^{35,36}$

Smoking has been known to influence VC in normal persons. Expected results include decline in FEV1, FVC and FEV1/FVC ratio. ${ }^{37}$ It is interesting to note that in our study, there was no statistically significant difference in VC values between current and non/ex smokers. It can be surmised that smoking could have a preferential effect on some individuals, or that the effect of smoking on VC is overshadowed by the VC decline from tetraplegia alone.

Almenoff similarly found that there were greater contributions to the decline in lung function according to the level and completeness of injury than the effect of smoking alone, especially in the high quadriplegia group. ${ }^{38}$

The main limitation in our study is the absence of a control group to compare the decline of $\mathrm{VC}$ over the years in wheelchair bound persons with $\mathrm{VC}$ decline in tetraplegics, whether it is the effect of the tetraplegia or the confinement to a wheelchair that contributes to the decline. However, due to the retrospective nature of the study, documentation was not complete. It would also be desirable to have more frequent values of $\mathrm{VC}$ in order to do a proper time analysis of $\mathrm{VC}$ trend over time. Future prospective studies will be needed to address these issues.

Another criticism of this paper was that throughout the years, different people may have carried out the $\mathrm{VC}$ maneuver and introduced variability in the $\mathrm{VC}$ obtained. However, over the years, all the patients in this study were supervised closely by the attending physician, one of the authors of this paper, who personally instructed and supervised the procedures. The same equipment was used both in the wards and on an outpatient basis, and this was periodically updated and checked.

A third criticism was the lack of mention of the percentage of predicted VC for every patient in this study. This was due to the fact that information on the patient's height was not always available due to the retrospective nature of this study. However, this information, although nice to have, is not absolutely necessary in comparing the $\mathrm{VC}$ trend over time.

\section{Conclusion}

Vital capacity in tetraplegics decrease with age as well as duration of spinal cord injury, especially more than 10 years post injury. This could be due to synergistic or additive effect of age and of duration of SCI on the decline in pulmonary function. 


\section{References}

1 Bergofsky EH. Mechanisms for respiratory insufficiency after cervical cord injury. Ann Intern Med 1964; 61: 435- 447.

2 Estenne M, De Troyer A. Relationship between respiratory muscle electromyogram and rib cage motion in tetraplegia. $\mathrm{Am}$ Rev Resp Dis 1985; 132: 53 - 59.

3 Roth EJ et al. Pulmonary function testing in spinal cord injury: correlation with vital capacity. Paraplegia 1995; 33: $454-457$.

4 American Thoracic Society. Medical Section of the American Lung Association Standardization of Spirometry 1994 Update. Am J Respir Crit Care Med 1995; 152: 1107-1136.

5 Ledsome JR, Sharp JM. Pulmonary function in acute cervical cord injury. Am Rev Resp Dis 1987; 124: $41-44$.

6 Coll JR, Frankel HL, Chalifue SW, Whiteneck GG. Evaluating neurological group homogeneity in assessing the mortality risk for people with spinal cord injuries. Spinal Cord 1998; 35: 275 279.

7 Donovan WH. Profile of the Institute of Rehabilitation and Research. Spinal Cord 1997; 35: 565-568.

8 Le CT, Price M. Survival from spinal cord injury. J Chron Dis 1982; 31: 487-492.

9 Carter RE. Respiratory management, including ventilator care in tetraplegia and diaphragmatic pacing. In: Frankel HL (ed). Handbook of Clinical Neurology, vol 17, Elsevier Science Publishers B.V., 1992, pp 261-274.

10 Moulton A, Silver JR. Chest movements in patients with traumatic injuries of the cervical cord. Clin Sci 1970; 39: $407-$ 422.

11 De Troyer A, Estenne M, Vinken W. Rib cage motions and muscle use in high tetraplegics. Am Rev Resp Dis 1986; 133: 1115.

12 Stone DJ, Keltz H. The effect of respiratory muscle dysfunction on pulmonary function. Am Rev Respir Dis 1963; 88: 621-629.

13 DeTroyer A, Heilporn A. Respiratory mechanics in quadriplegia. The respiratory function of the intercostal muscles. Am Rev Respir Dis 1980; 122: $591-600$.

14 Kelly BJ, Luce JM. The diagnosis and management of neuromuscular diseases causing respiratory failure. Chest 1991 ; 99: $1485-1494$.

15 Estenne $\mathrm{M}$ et al. The effect of pectoralis muscle training in tetraplegic subjects. Am Rev Resp Dis 1989; 139: 1218-1222.

16 Kenney RA. Physiology of Aging, 2nd edn. Chicago, Year Book, 1989.

17 DeMello D, Reid LM. Development, growth and aging of the lung. In Saldana MJ (ed). Pathology of Pulmonary Disease. Philadelphia, JB Lippincott, 1994.

18 Grimm DR, Spungen AM, Bauman WA, Almenoff PL. Day to day variability in spirometry testing in individuals with spinal cord injury. J Spinal Cord Med 1996; 19: 129.

19 McMichan JC, Michel L, Westbrook P. Pulmonary dysfunction following traumatic quadriplegia. JAMA 1981; 243: 528-531.

20 Rutchik A et al. Resistive inspiratory muscle training in subjects with chronic cervical spinal injury. Arch Phys Med Rehab 1998; 79: $293-297$.
21 Gross D et al. The effect of training on strength and endurance of the diaphragm in quadriplegia. Am J Med 1980; 68: 27-35.

22 Huldtgren AC, Fugl-Meyer AR, Jonasson E, Bake B. Ventilatory dysfunction and respiratory rehabilitation in post-traumatic quadriplegia. Eur J Respir Dis 1980; 61: 347-356.

23 Carter RE. Experiences with high tetraplegics. Paraplegia 1979; 17: $140-146$

24 Hoffman MD. Cardiorespiratory fitness and training in quadriplegics and paraplegia. Sports Med 1986; 3: $312-330$.

25 Scanlon PD et al. Respiratory mechanics in acute quadriplegia: lung and chest wall compliance and dimensional changes during respiratory manoeuvres. Am Rev Respir Dis 1989; 139: 615-620.

26 Bach JR, Alba AS. Noninvasive options for ventilatory support of the traumatic high level quadriplegia. Chest 1990; 98: $613-$ 619.

27 Bach JR, Wang TG. Pulmonary function and sleep disordered breathing in patients with traumatic paraplegia: A longitudinal study. Arch Phys Med Rehab 1994; 75: 279-284.

28 De Troyer A, Borenstein S, Ciordier R. Analysis of lung volume restriction in patients with respiratory muscle weakness. Thorax 1980; 35: $603-610$.

29 Needham CD, Rogan MC, McDonald L. Normal standards for lung volumes, intrapulmonary gas mixing and maximal breathing capacity. Thorax 1954; 9: 313-325.

30 Fisher AD. Poliomyelitis: later respiratory complications and management. Orthopedics 1985; 8: $891-894$.

31 Menter RM, Hudson LM. Effects of age at injury and the aging process. In: Stover SL, DeLisa JA, Whiteneck GG (eds). Spinal cord injury - Clinical outcomes from the model systems. Aspen Publishers, Gaithersburg, Maryland, 1995; pp 272-288.

32 Whiteneck GG et al. Mortality, morbidity and psychosocial outcomes of persons spinal cord injured more than 20 years ago. Paraplegia 1992; 30: 617-630.

33 Hsu S, Dreisbach JN, Charlifue SW, English GM. Glottic and tracheal stenosis in spinal cord injured patients. Paraplegia 1987; 25: $136-148$

34 DeVivo M, Black K, Stover S. Causes of death during the first 12 years after spinal cord injury. Arch Phys Med Rehabil 1993; 74: $248-254$

35 Klefback B et al. Obstructive sleep apneas in relation to severity of cervical spinal cord injury. Spinal Cord 1998; 36: 621-628.

36 Sakjov D et al. Sleep apnea related hypoxia is associated with cognitive disturbances in patients with tetraplegia. Spinal Cord 1998; 34: $231-239$.

37 Aparici M, Fernandez Gonzalez AL, Algeria E. Respiratory function tests. Differences between smokers and non-smokers. Effects of withdrawal. Rev Clin Esp 1993; 192: 169-172.

38 Almenoff PL et al. Pulmonary function survey in spinal cord injury: influences of smoking and level and completeness of injury. Lung 1995; 173: 297-306. 